\title{
Kybernetika
}

\section{Mehdi Rahimi}

\section{A local approach to $g$-entropy}

Kybernetika, Vol. 51 (2015), No. 2, 231-245

Persistent URL: http://dml.cz/dmlcz/144294

\section{Terms of use:}

(C) Institute of Information Theory and Automation AS CR, 2015

Institute of Mathematics of the Czech Academy of Sciences provides access to digitized documents strictly for personal use. Each copy of any part of this document must contain these Terms of use.

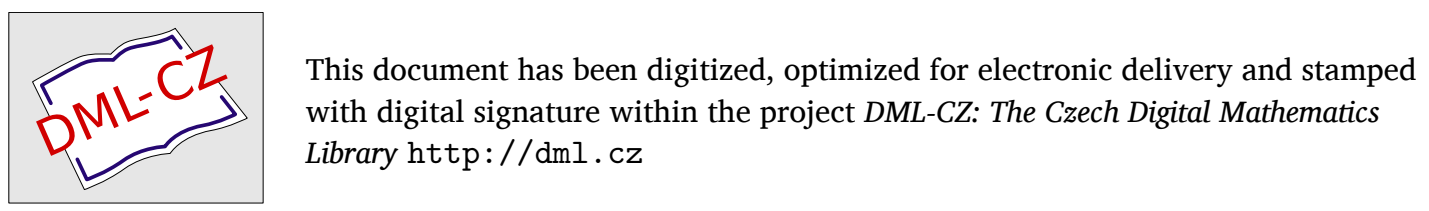




\title{
A LOCAL APPROACH TO $g$-ENTROPY
}

\author{
MEHDI RAHIMI
}

In this paper, a local approach to the concept of $g$-entropy is presented. Applying the Choquet's representation Theorem, the introduced concept is stated in terms of $g$-entropy.

Keywords: fuzzy entropy, $g$-entropy, local entropy

Classification: 28D20, 28E10

\section{INTRODUCTION}

The fuzzy entropy of dynamical systems is studied extensively [3, 4, 5, 6, 7, 8, 9, 10, 12, 15, 17, 19, 20. It is based on the idea of replacing partitions, in the classical ergodic theory, by fuzzy partitions.

The concept of $g$-entropy, as a generalized form of the fuzzy entropy, is studied by Mesiar and Rybarik [12, 17, 20] and its Hudetz correction is discussed in [16. It is based on an increasing bijective map $g:[0, \infty] \rightarrow[0, \infty]$ such that $g(0)=0$ and $g(1)=1$.

In classical ergodic theory, local studies of entropy of dynamical systems is studied extensively [1, 11, 13, 21, 23. A local study of the fuzzy entropy of dynamical systems, in the sense of Dumitrescu, was arranged in [14.

The main goal of this paper is to apply the method used in [14] to present a local approach to $g$-entropy of a dynamical system. This approach is of topological nature, in the sense that, the set of all $g$-decomposable measures is equipped by a topology which provides the requirements of the Choquet's representation Theorem. It enables us to state the introduced entropy in this paper in terms of the $g$-entropy [12, 17, 20].

In section 2 , we recall some preliminary concepts. In section 3 , we define a topology on the set of invariant measures and prove some results which leads to the $g$-ergodic decomposition of invariant measures. In section 4 , a new version of $g$-entropy, in a local approach, is defined. Finally, the new quantity is stated in terms of $g$-entropy.

\section{PRELIMINARY CONCEPTS}

In this section, we provide some known facts which will be used in the remaining of the paper. From now on, $g:[0, \infty] \rightarrow[0, \infty]$ is an increasing bijective function such that $g(0)=0$ and $g(1)=1$. The following definitions are mainly from [16].

DOI: 10.14736/kyb-2015-2-0231 
A fuzzy $\sigma$-algebra $\mathcal{F}$ on $X$ is a collection of fuzzy subsets of $X$, i. e., functions $f$ : $X \rightarrow[0,1]$, satisfying the following conditions:

(i) $1_{X} \in \mathcal{F}$.

(ii) If $f, g \in \mathcal{F}$ then $f . g \in \mathcal{F}$ and $(f-g)^{+} \in \mathcal{F}$ where $(f-g)^{+}(x):=\max \{(f-g)(x), 0\}$ for all $x \in X$.

(iii) If $\left\{f_{n}\right\}_{n=1}^{\infty} \subset \mathcal{F}$ then $\bigvee_{n=1}^{\infty} f_{n} \in \mathcal{F}$ where $\bigvee_{n=1}^{\infty} f_{n}:=\min \left\{\sum_{n=1}^{\infty} f_{n}, 1\right\}$

A function $m^{*}: \mathcal{F} \rightarrow[0, \infty)$ is called a fuzzy measure, if

(i) $m^{*}\left(0_{X}\right)=0$.

(ii) $m^{*}\left(\bigvee_{n=1}^{\infty} f_{n}\right)=\sum_{n=1}^{\infty} m^{*}\left(f_{n}\right)$, whenever $\left\{f_{n}\right\}_{n=1}^{\infty} \subset \mathcal{F}$ and $\sum_{n=1}^{\infty} f_{n} \leq 1$.

A $g$-decomposable measure on $\mathcal{F}$ is a mapping $m: \mathcal{F} \rightarrow[0,1]$ such that $m\left(1_{X}\right)=1$, $m\left(0_{X}\right)=0$ and

$$
m\left(g^{-1}\left(\sum_{n=1}^{\infty} g \circ f_{n}\right)\right)=g^{-1}\left(\sum_{n=1}^{\infty} g\left(m\left(f_{n}\right)\right)\right)
$$

whenever $f_{n} \in \mathcal{F}(n=1,2,3, \ldots)$ are such that $\sum_{n=1}^{\infty} g \circ f_{n} \leq 1$.

If $m$ is a $g$-decomposable measure on $\mathcal{F}$ then the function

$$
m^{*}:=g \circ m \circ g^{-1}
$$

is a fuzzy measure on $\mathcal{F}$.

A family $\xi=\left\{f_{1}, f_{2}, \ldots, f_{k}\right\}$ of members of $\mathcal{F}$ is a $g$-fuzzy partition of $X$, if $\sum_{i=1}^{k} g \circ$ $f_{i}=1$ on $X$. When $g(x)=x$, a $g$-fuzzy partition is nothing but a fuzzy partition, i. e., a family $\xi=\left\{f_{1}, f_{2}, \ldots, f_{k}\right\}$ such that $\sum_{i=1}^{k} f_{i}=1$ on $X$. Note that, if $\xi=\left\{f_{1}, f_{2}, \ldots, f_{k}\right\}$ is a $g$-fuzzy partition then $g(\xi)=\left\{g \circ f_{1}, g \circ f_{2}, \ldots, g \circ f_{k}\right\}$ is a fuzzy partition.

The $g$-entropy $H_{m, g}$ of a $g$-fuzzy partition $\xi=\left\{f_{1}, f_{2}, \ldots, f_{k}\right\}$ is defined by the formula

$$
H_{m, g}(\xi)=g^{-1}\left(\sum_{i=1}^{k} g\left(\Phi\left(m\left(f_{i}\right)\right)\right)\right)
$$

where $\Phi=g^{-1} \circ \phi \circ g$ and $\phi(x)=-x \log x$ for $x \neq 0, \phi(0)=0$. Hence

$$
H_{m, g}(\xi)=g^{-1}\left(\sum_{i=1}^{k} \phi\left(m^{*}\left(g\left(f_{i}\right)\right)\right)\right) .
$$

The joint of two $g$-fuzzy partitions $\xi=\left\{f_{1}, f_{2}, \ldots, f_{k}\right\}$ and $\eta=\left\{h_{1}, h_{2}, \ldots, h_{t}\right\}$ is defined by

$$
\xi \vee \eta=\left\{g^{-1}\left(\left(g \circ f_{i}\right)\left(g \circ h_{j}\right)\right): i=1, \ldots, k, j=1, \ldots, t\right\} .
$$

Note that, in the case of $g(x)=x$ for all $x \in X$, if $\xi$ and $\eta$ are two fuzzy partitions then

$$
\xi \vee \eta=\left\{f_{i} h_{j}: i=1, \ldots, k, j=1, \ldots, t\right\} .
$$


Suppose that $T:(X, \mathcal{B}) \rightarrow(X, \mathcal{B})$ is a measurable mapping and $\mathcal{F}$ is a fuzzy $\sigma$-algebra of $\mathcal{B}$-measurable fuzzy subsets of $X$. For a $g$-decomposable measure $m$, and a $g$-fuzzy partition $\xi$, the $g$-entropy of $T$ with respect to $\xi$ is defined as:

$$
h_{m, g}(T, \xi):=\lim _{n \rightarrow \infty} g^{-1}\left(\frac{1}{n} g\left(H_{m, g}\left(\bigvee_{i=0}^{n-1} T^{-i} \xi\right)\right)\right)
$$

where $T^{-i} \xi=\left\{f_{1} \circ T^{i}, f_{2} \circ T^{i}, \ldots, f_{k} \circ T^{i}\right\}$.

Finally, the $g$-entropy of $T$ is defined by:

$$
h_{m, g}(T):=\sup _{\xi} h_{m, g}(T, \xi)
$$

where the supremum is taken over all $g$-fuzzy partitions. Note that, the fuzzy entropy $h_{m^{*}}(T)$ can be obtained putting $g(u)=u, u \in[0,1]$. We recall that, the entropy of a fuzzy partition $\xi=\left\{f_{1}, f_{2}, \ldots, f_{k}\right\}$ is given by $H_{m^{*}}(\xi)=-\sum_{i=1}^{k} m^{*}\left(f_{i}\right) \log m^{*}\left(f_{i}\right)$. The entropy of a dynamical system $T$ with respect to the fuzzy entropy $\xi$ is given by

$$
h_{m^{*}}(T, \xi)=\lim _{n \rightarrow \infty} \frac{1}{n} H_{m^{*}}\left(\bigvee_{i=0}^{n-1} T^{-i} \xi\right)
$$

and the fuzzy entropy of $T$ is given by

$$
h_{m^{*}}(T)=\sup _{\xi} h_{m^{*}}(T, \xi)
$$

where the supremum is taken over all fuzzy partitions $\xi$.

The following theorem makes a connection between the concept of $g$-entropy and fuzzy entropy.

Theorem 2.1. Let $T, g$ and $\xi$ be as above and $m^{*}$ be a fuzzy measure defined by (1). Then

(i) $H_{m, g}(\xi)=g^{-1}\left(H_{m^{*}}(g(\xi))\right)$;

(ii) $h_{m, g}(T, \xi)=g^{-1}\left(h_{m^{*}}(T, g(\xi))\right)$;

(iii) $h_{m, g}(T)=g^{-1}\left(h_{m^{*}}(T)\right)$.

Proof. See [18] Proposition 10.6.6, Proposition 10.6.11 and Theorem 10.6.13.

Theorem 2.2. (Butnariu and Klement 2]) Suppose that $\mathcal{F}$ is a fuzzy $\sigma$-algebra on $X$ and $m^{*}$ is a fuzzy measure on $\mathcal{F}$. Let $\mathfrak{B}=\left\{A \subset X: \chi_{A} \in \mathcal{F}\right\}$ and let $\mu_{m^{*}}: \mathfrak{B} \rightarrow \mathbb{R}$ be defined by $\mu_{m^{*}}(A):=m^{*}\left(\chi_{A}\right)$. Then every $f \in \mathcal{F}$ is $\mathfrak{B}$-measurable and $m^{*}(f)=$ $\int_{X} f \mathrm{~d} \mu_{m^{*}}$ for all $f \in \mathcal{F}$. 
Corollary 2.3. Let $\mathcal{F}$ be a fuzzy $\sigma$-algebra on $X$ and $m$ is a $g$-decomposable measure on $X$. Then $m^{*}:=g \circ m \circ g^{-1}$ is a fuzzy measure on $\mathcal{F}$ and $\mu_{m^{*}}$ is a measure on the $\sigma$-algebra $\mathfrak{B}=\left\{A \subset X: \chi_{A} \in \mathcal{F}\right\}$ such that

$$
m(f)=g^{-1}\left(\int_{X} g \circ f \mathrm{~d} \mu_{m^{*}}\right)
$$

for all $f \in \mathcal{F}$.

\section{TOPOLOGY ON INVARIANT MEASURES}

In this section, let $T: X \rightarrow X$ be a continuous map on a compact metric space $X$. Let $\mathcal{F} \subset[0,1]^{X}$ be the family of all Borel measurable maps $f: X \rightarrow[0,1]$. Then the corresponding $\sigma$-algebra given by Theorem 2.2 is indeed the $\sigma$-algebra of Borel sets of $X$. The set of all fuzzy measures $m: \mathcal{F} \rightarrow[0, \infty]$, satisfying $m\left(1_{X}\right)=1$ is denoted by $M^{*}(X)$. Let $g:[0, \infty] \rightarrow[0, \infty]$ be an increasing bijective function such that $g(0)=0$ and $g(1)=1$. The set of $g$-invariant measures of $T$ is defined by

$$
M_{g}^{*}(X, T):=\left\{m \in M^{*}(X): m\left(g^{-1} \circ f \circ T\right)=m\left(g^{-1} \circ f\right) \forall f \in \mathcal{F}\right\} .
$$

A $g$-decomposable measure $m \in M_{g}^{*}(X, T)$ is said to be $g$-ergodic, if the following implication holds for all $f \in \mathcal{F}$ :

$$
f \circ T=f \Rightarrow m\left(g^{-1} \circ f\right)=0 \text { or } 1 .
$$

The set of all $g$-fuzzy ergodic measures of $T$ is denoted by $E_{g}^{*}(X, T)$.

The following lemma connects the $g$-invariant and $g$-ergodic measures to the classical invariant and ergodic measures.

Lemma 3.1. Let $T: X \rightarrow X$ be a continuous map on a compact metric space $X$. Let $M(X, T)$ and $E(X, T)$ be the set of invariant and ergodic measures of $T$ in the classical sense respectively. Let $m^{*}$ be a fuzzy measure defined by (1). Then

(i) $m \in M_{g}^{*}(X, T)$ if and only if $\mu_{m^{*}} \in M(X, T)$.

(ii) If $m \in E_{g}^{*}(X, T)$ then $\mu_{m^{*}} \in E(X, T)$.

Proof. (i) Let $m \in M_{g}^{*}(X, T)$. For any Borel set $A$, if $f=\chi_{A}$ then $m\left(g^{-1} \circ \chi_{A} \circ T\right)=$ $m\left(g^{-1} \circ \chi_{A}\right)$, therefore

$$
\begin{aligned}
\mu_{m^{*}}\left(T^{-1}(A)\right) & =\int_{X} \chi_{T^{-1}(A)} \mathrm{d} \mu_{m^{*}} \\
& =\int_{X} \chi_{A} \circ T \mathrm{~d} \mu_{m^{*}} \\
& =m^{*}\left(\chi_{A} \circ T\right) \\
& =g\left(m\left(\left(g^{-1} \circ \chi_{A}\right) \circ T\right)\right) \\
& =g\left(m\left(g^{-1} \circ \chi_{A}\right)\right) \\
& =m^{*}\left(\chi_{A}\right) \\
& =\mu_{m^{*}}(A) .
\end{aligned}
$$


This means $\mu_{m^{*}} \in M(X, T)$.

Conversely, let $\mu_{m^{*}} \in M(X, T)$. For any characteristic function $f=\chi_{A}$, where $A$ is a Borel set, we have

$$
\begin{aligned}
m\left(g^{-1} \circ f \circ T\right) & =m\left(g^{-1} \circ \chi_{A} \circ T\right) \\
& =g^{-1}\left(m^{*}\left(\chi_{A} \circ T\right)\right) \\
& =g^{-1}\left(\int_{X} \chi_{A} \circ T \mathrm{~d} \mu_{m^{*}}\right) \\
& =g^{-1}\left(\int_{X} \chi_{T^{-1}(A)} \mathrm{d} \mu_{m^{*}}\right) \\
& =g^{-1}\left(\mu_{m^{*}}\left(T^{-1}(A)\right)\right) \\
& =g^{-1}\left(\mu_{m^{*}}(A)\right) \\
& =g^{-1}\left(m^{*}\left(\chi_{A}\right)\right) \\
& =m\left(g^{-1} \circ \chi_{A}\right) \\
& =m\left(g^{-1} \circ f\right),
\end{aligned}
$$

which gives the result for characteristic functions. Moreover, if $f=\sum_{i=1}^{k} c_{i} \chi_{A_{i}}$ is a simple function where $c_{i}, i=1,2, \ldots, k$, are different real numbers and $A_{i}, i=1,2, \ldots, k$, are pairwise disjoint Borel measurable, then

$$
\begin{aligned}
m\left(g^{-1} \circ f \circ T\right) & =g^{-1}\left(m^{*}(f \circ T)\right) \\
& =g^{-1}\left(\int_{X} f \circ T \mathrm{~d} \mu_{m^{*}}\right) \\
& =g^{-1}\left(\int_{X} \sum_{i=1}^{k} c_{i} \chi_{A_{i}} \mathrm{~d} \mu_{m^{*}}\right) \\
& =g^{-1}\left(\sum_{i=1}^{k} c_{i} m^{*}\left(\chi_{A_{i}} \circ T\right)\right) \\
& =g^{-1}\left(\sum_{i=1}^{k} c_{i} m^{*}\left(\chi_{A_{i}}\right)\right) \\
& =g^{-1}\left(\sum_{i=1}^{k} c_{i} \int_{X} \chi_{A_{i}} \mathrm{~d} \mu_{m^{*}}\right) \\
& =g^{-1}\left(\int_{X}^{k} c_{i=1} \chi_{A_{i}} \mathrm{~d} \mu_{m^{*}}\right) \\
& =g^{-1}\left(m^{*}(f)\right) \\
& \left.=m^{-1} \circ f\right),
\end{aligned}
$$

which gives the result for simple functions.

Finally, let $f \in \mathcal{F}$. Let $\left\{f_{n}\right\}_{n=1}^{\infty}$ be a sequence of simple functions such that $0 \leq f_{1} \leq$ 
$f_{2} \leq f_{3} \leq \ldots$ and $f_{n} \nearrow f$. Applying Monotone Convergence Theorem we will have

$$
\begin{aligned}
m\left(g^{-1} \circ f \circ T\right) & =g^{-1}\left(m^{*}(f \circ T)\right) \\
& =g^{-1}\left(\int_{X} f \circ T \mathrm{~d} \mu_{m^{*}}\right) \\
& =g^{-1}\left(\lim _{n \rightarrow \infty} \int_{X} f_{n} \circ T \mathrm{~d} \mu_{m^{*}}\right) \\
& =g^{-1}\left(\lim _{n \rightarrow \infty} m^{*}\left(f_{n} \circ T\right)\right) \\
& =g^{-1}\left(\lim _{n \rightarrow \infty} m^{*}\left(f_{n}\right)\right) \\
& =g^{-1}\left(\lim _{n \rightarrow \infty} \int_{X} f_{n} \mathrm{~d} \mu_{m^{*}}\right) \\
& =g^{-1}\left(\int_{X} f \mathrm{~d} \mu_{m^{*}}\right) \\
& =g^{-1}\left(m^{*}(f)\right) \\
& =m\left(g^{-1} \circ f\right) .
\end{aligned}
$$

It completes the proof of (i).

(ii) Let $m \in E_{g}^{*}(X, T)$. If $A$ is a Borel measurable set such that $T^{-1}(A)=A$ then $\chi_{T^{-1}(A)}=\chi_{A}$ or equivalently $\chi_{A} \circ T=\chi_{A}$, therefore $m\left(g^{-1} \circ \chi_{A}\right)=0$ or 1 , that is $g^{-1}\left(m^{*}\left(\chi_{A}\right)\right)=0$ or 1 which means $g^{-1}\left(\mu_{m^{*}}(A)\right)=0$ or 1 , consequently $\mu_{m^{*}}(A)=0$ or 1 , since $g$ is injective. It proves that $\mu_{m^{*}} \in E(X, T)$.

In the following, $M^{*}(X)$ is equipped by a topology in a natural way.

Definition 3.2. The $\mathrm{w}^{*}$-topology on $M^{*}(X)$ is the smallest topology making each of the maps $m^{*} \mapsto \int_{X} f \mathrm{~d} \mu_{m^{*}}(f \in C(X))$ continuous. A basis is given by the collection of all sets of the form

$$
V_{m_{0}^{*}}\left(f_{1}, \ldots, f_{k} ; \epsilon\right)=\left\{m^{*} \in M^{*}(X):\left|\int_{X} f_{i} \mathrm{~d} \mu_{m^{*}}-\int_{X} f_{i} \mathrm{~d} \mu_{m_{0}^{*}}\right|<\epsilon, 1 \leq i \leq k\right\}
$$

where $m_{0}^{*} \in M^{*}(X), k \geq 1, f_{i} \in C(X)$ and $\epsilon>0$.

By the correspondence given in Theorem 2.2, the previous topology is indeed the weak* topology defined on $M(X)$ in the classical case. So all of the properties of the weak* topology defined on $M(X)$ is inherited to $M^{*}(X)$. We summarize the most important properties of $M^{*}(X)$ in the following theorem.

Theorem 3.3. Let $X$ be a compact metrizable space and let $T: X \rightarrow X$ be continuous. Let $\mathcal{F} \subset[0,1]^{X}$ be the $\sigma$-algebra of all Borel measurable maps $f: X \rightarrow[0,1]$. Then

(i) The space $M^{*}(X)$ is metrizable in the $\mathrm{w}^{*}$-topology. If $\left\{f_{n}\right\}_{n=1}^{\infty}$ is a dense subset of $C(X)$ then

$$
D\left(m, m^{\prime}\right)=\sum_{n=1}^{\infty} \frac{\left|\int_{X} f_{n} \mathrm{~d} \mu_{m^{*}}-\int_{X} f_{n} \mathrm{~d} \mu_{m^{\prime *}}\right|}{2^{n}\left\|f_{n}\right\|}
$$


is a metric on $M^{*}(X)$ giving the $\mathrm{w}^{*}$-topology.

(ii) For $m_{n}, m \in M^{*}(X)(n \geq 1), m_{n} \rightarrow m$ if and only if $\int_{X} f \mathrm{~d} \mu_{m_{n}^{*}} \rightarrow \int_{X} f \mathrm{~d} \mu_{m^{*}}$ for all $f \in C(X)$.

(iii) $M_{g}^{*}(X, T)$ is a compact subset of $M^{*}(X)$.

(iv) $M_{g}^{*}(X, T)$ is convex.

(v) $\operatorname{ext}\left(M_{g}^{*}(X, T)\right)=E_{g}^{*}(X, T)$.

Proof. See 24] Theorems 6.4 and 6.10.

Definition 3.4. Suppose that $Y$ is a non-empty compact subset of a locally convex space $E$, and let $\tau$ be a probability measure on $Y$. A point $x$ in $E$ is said to be represented by $\tau$ if $\Phi(x)=\int_{Y} \Phi \mathrm{d} \tau$ for every continuous linear functional $\Phi$ on $E$.

Theorem 3.5. (Choquet) Suppose that $Y$ is a metrizable compact convex subset of a locally convex space $E$, and that $x_{0}$ is an element of $Y$. Then there exists a probability measure $\tau$ on $Y$ which represents $x_{0}$ and is supported by the extreme points of $Y$.

See Phelps 22 for a proof of Choquet's Theorem.

By Theorem 3.3 $M^{*}(X)$ is a compact metrizable space and $M_{g}^{*}(X, T)$ is a compact metrizable convex set with the extreme points $E_{g}^{*}(X, T)$. So applying the Choquet's Theorem we will have the following corollary.

Corollary 3.6. For any $m \in M_{g}^{*}(X, T)$ there exists a unique probability measure $\tau$ on the Borel subsets of the compact metrizable space $M_{g}^{*}(X, T)$ such that $\tau\left(E_{g}^{*}(X, T)\right)=1$ and

$$
\int_{X} f(x) \mathrm{d} \mu_{m^{*}}(x)=\int_{E_{g}^{*}(X, T)}\left(\int_{X} f(x) \mathrm{d} \mu_{\nu^{*}}(x)\right) \mathrm{d} \tau(\nu)
$$

for every bounded measurable function $f: X \rightarrow \mathbb{R}$.

In particular, if $f \in \mathcal{F}$ then the previous equality is indeed

$$
m^{*}(f)=\int_{E_{g}^{*}(X, T)} \nu^{*}(f) \mathrm{d} \tau(\nu) .
$$

Since $m^{*}=g \circ m \circ g^{-1}$ then

$$
g \circ m \circ g^{-1}(f)=\int_{E_{g}^{*}(X, T)} g \circ \nu \circ g^{-1}(f) \mathrm{d} \tau(\nu) .
$$

Replacing $f$ by $g \circ f$ in the previous relation we will have

$$
m(f)=g^{-1}\left(\int_{E_{g}^{*}(X, T)} g(\nu(f)) \mathrm{d} \tau(\nu)\right) .
$$

Under the assumptions of Corollary 3.6 we write $m=\int_{E_{g}^{*}(X, T)} \nu \mathrm{d} \tau(\nu)$ and it is called the $g$-ergodic decomposition of $m$. 


\section{LOCAL $g$-ENTROPY}

In this section, $T: X \rightarrow X$ is a continuous map on a compact metric space $X$ and $\mathcal{F}$ is the $\sigma$-algebra of Borel measurable maps $f: X \rightarrow[0,1]$. As before, let $g:[0, \infty] \rightarrow[0, \infty]$ be an increasing function such that $g(0)=0$ and $g(1)=1$.

Definition 4.1. For $x \in X$ and $f \in \mathcal{F}$, define

$$
\omega_{g}(T, x, f):=g^{-1}\left(\limsup _{n \rightarrow \infty} \frac{1}{n} \sum_{k=0}^{n-1}\left((g \circ f) \circ T^{k}\right)(x)\right) .
$$

We write $\omega(T, x, f)$ for the special case $g(x)=x$, indeed

$$
\omega(T, x, f)=\limsup _{n \rightarrow \infty} \frac{1}{n} \sum_{i=0}^{n-1}\left(f \circ T^{i}\right)(x) .
$$

Definition 4.2. Let $\xi=\left\{f_{1}, f_{2}, \ldots, f_{k}\right\}$ be a $g$-partition and $x \in X$. Define

$$
\begin{aligned}
\Omega_{g}(T, x, \xi) & :=g^{-1}\left(\sum_{i=1}^{k} g\left(\Phi\left(\omega_{g}\left(T, x, f_{i}\right)\right)\right)\right) \\
& =g^{-1}\left(\sum_{i=1}^{k} \phi\left(g\left(\omega_{g}\left(T, x, f_{i}\right)\right)\right)\right) \\
& =g^{-1}\left(-\sum_{i=1}^{k} g\left(\omega_{g}\left(T, x, f_{i}\right)\right) \log g\left(\omega_{g}\left(T, x, f_{i}\right)\right)\right) .
\end{aligned}
$$

If $\xi=\left\{f_{1}, f_{2}, \ldots, f_{k}\right\}$ is a fuzzy partition, the special case $g(x)=x$ of the definition 4.2 is given by

$$
\Omega(T, x, \xi)=-\sum_{i=1}^{k} \omega\left(T, x, f_{i}\right) \log \omega\left(T, x, f_{i}\right)=\sum_{i=1}^{k} \phi\left(\omega\left(T, x, f_{i}\right)\right) .
$$

Definition 4.3. Let $\xi=\left\{f_{1}, f_{2}, \ldots, f_{k}\right\}$ be a $g$-partition and $x \in X$. Define

$$
\mathcal{H}_{g}(T, x, \xi):=\limsup _{n \rightarrow \infty} g^{-1}\left(\frac{1}{n} g\left(\Omega_{g}\left(T, x, \bigvee_{i=0}^{n-1} T^{-i} \xi\right)\right)\right)
$$

Setting $g(x)=x$, in Definition 4.3 will result in the following:

$$
\mathcal{H}(T, x, \xi)=\limsup _{n \rightarrow \infty} \frac{1}{n} \Omega\left(T, x, \bigvee_{i=0}^{n-1} T^{-i} \xi\right)
$$

where $\xi=\left\{f_{1}, f_{2}, \ldots, f_{k}\right\}$ is a fuzzy partition. 
Definition 4.4. Let $\xi$ be a $g$-partition and $m \in M_{g}^{*}(X, T)$. Define

$$
h_{m, g}^{*}(T, \xi):=\int_{X} \mathcal{H}_{g}(T, x, \xi) \mathrm{d} \mu_{m^{*}}(x)
$$

and

$$
h_{m, g}^{*}(T):=\sup _{\xi} h_{m, g}^{*}(T, \xi)
$$

where the supremum is taken over all $g$-fuzzy partitions.

If $m^{*}$ is a fuzzy measure and $\xi$ is a fuzzy partition, the special case $g(x)=x$ in Definition 4.4 results in the following:

$$
h_{m^{*}}^{*}(T, \xi)=\int_{X} \mathcal{H}(T, x, \xi) \mathrm{d} \mu_{m^{*}}(x)
$$

and

$$
h_{m^{*}}^{*}(T)=\sup _{\xi} h_{m^{*}}^{*}(T, \xi)
$$

where the supremum is taken over all fuzzy partitions.

In [14], the properties of the previous quantities in the case of $g(x)=x$ for all $x \in X$, are discussed.

Theorem 4.5. Let $x \in X$ and $\xi$ be a $g$-fuzzy partition. Then

(i) $\Omega_{g}(T, x, \xi)=g^{-1}(\Omega(T, x, g(\xi)))$;

(ii) $\mathcal{H}_{g}(T, x, \xi)=g^{-1}(\mathcal{H}(T, x, g(\xi)))$;

(iii) If $g$ is convex then $h_{m, g}^{*}(T) \leq g^{-1}\left(h_{m^{*}}^{*}(T)\right)$.

Proof. (i) First note that, by Definition 4.1. $g\left(\omega_{g}(T, x, f)\right)=\omega(T, x, f)$. Now, the result follows directly from Definition 4.2 .

(ii) follows from (i) and the equality $g\left(\bigvee_{i=0}^{n-1} T^{-i} \xi\right)=\bigvee_{i=0}^{n-1} T^{-i} g(\xi)$.

(iii) For a $g$-fuzzy partition $\xi$, applying part (ii) and Jensen's inequality we will have

$$
\begin{aligned}
h_{m, g}^{*}(T, \xi) & =\int_{X} \mathcal{H}_{g}(T, x, \xi) \mathrm{d} \mu_{m^{*}}(x) \\
& =\int_{X} g^{-1}(\mathcal{H}(T, x, g(\xi))) \mathrm{d} \mu_{m^{*}}(x) \\
& \leq g^{-1}\left(\int_{X} \mathcal{H}(T, x, g(\xi)) \mathrm{d} \mu_{m^{*}}(x)\right) \\
& =g^{-1}\left(h_{m^{*}}(T, g(\xi))\right) \\
& \leq g^{-1}\left(h_{m^{*}}(T)\right),
\end{aligned}
$$

where the last inequality holds because $g^{-1}$ is also increasing. Finally, taking supremum over all $g$-fuzzy partitions we will get the result. 
Theorem 4.6. Suppose that $T: X \rightarrow X$ is a continuous map on a compact metric space $X$. If $\xi, \eta$ are $g$-fuzzy partitions and $x \in X$ then

(i) If $\xi \leq \eta$ then $\Omega_{g}(T, x, \xi) \leq \Omega_{g}(T, x, \eta)$.

(ii) If $\xi \leq \eta$ then $\mathcal{H}_{g}(T, x, \xi) \leq \mathcal{H}_{g}(T, x, \eta)$.

Proof. Let $\xi=\left\{f_{i}\right\}$ and $\eta=\left\{h_{j}\right\}$ be two $g$-fuzzy partitions and assume, without loss of generality, that all fuzzy sets are such that $\omega_{g}(T, x, f) \neq 0$. (Since if $\xi=\left\{f_{1}, f_{2}, \ldots, f_{k}\right\}$ with $\omega_{g}(T, x, f)>0$ for $1 \leq i \leq r$ and $\omega_{g}(T, x, f)=0$ for $r<i \leq k$ we can replace $\xi$ by $\left.\left\{f_{1} \vee f_{2} \vee \ldots \vee f_{k}\right\}\right)$.

(i) Since $\xi \leq \eta$ we have $\xi \vee \eta=\eta$. By definition we obtain

$$
\begin{aligned}
g\left(\Omega_{g}(T, x, \eta)\right) & =g\left(\Omega_{g}(T, x, \xi \vee \eta)\right) \\
& =\Omega(T, x, g(\xi \vee \eta)) \\
& =\Omega(T, x, g(\xi) \vee g(\eta)) \\
& =-\sum_{i, j} \omega\left(T, x,\left(g \circ f_{i}\right)\left(g \circ h_{j}\right)\right) \log \omega\left(T, x,\left(g \circ f_{i}\right)\left(g \circ h_{j}\right)\right) \\
& =-\sum_{i, j} \omega\left(T, x,\left(g \circ f_{i}\right)\left(g \circ h_{j}\right)\right) \log \frac{\omega\left(T, x,\left(g \circ f_{i}\right)\left(g \circ h_{j}\right)\right)}{\omega\left(T, x, g \circ f_{i}\right)} \omega\left(T, x, g \circ f_{i}\right) \\
& =-\sum_{i, j} \omega\left(T, x,\left(g \circ f_{i}\right)\left(g \circ h_{j}\right)\right) \log \frac{\omega\left(T, x,\left(g \circ f_{i}\right)\left(g \circ h_{j}\right)\right)}{\omega\left(T, x, g \circ f_{i}\right)} \\
& -\sum_{i, j} \omega\left(T, x,\left(g \circ f_{i}\right)\left(g \circ h_{j}\right)\right) \log \omega\left(T, x, g \circ f_{i}\right) \\
\geq & -\sum_{i, j} \omega\left(T, x,\left(g \circ f_{i}\right)\left(g \circ h_{j}\right)\right) \log \omega\left(T, x, g \circ f_{i}\right)
\end{aligned}
$$

where the last inequality holds since $\omega\left(T, x,\left(g \circ f_{i}\right)\left(g \circ h_{j}\right)\right) \leq \omega\left(T, x,\left(g \circ f_{i}\right)\right)$.

On the other hand, since

$$
\omega\left(T, x, g \circ f_{i}\right) \leq \sum_{j} \omega\left(T, x,\left(g \circ f_{i}\right)\left(g \circ h_{j}\right)\right)
$$

we conclude that

$$
-\sum_{i, j} \omega\left(T, x,\left(g \circ f_{i}\right)\left(g \circ h_{j}\right)\right) \log \omega\left(T, x, g \circ f_{i}\right) \geq-\sum_{i} \omega\left(T, x,\left(g \circ f_{i}\right)\right) \log \omega\left(T, x, g \circ f_{i}\right) .
$$

Combining (2) and (3) we will have

$$
g\left(\Omega_{g}(T, x, \eta)\right) \geq g\left(\Omega_{g}(T, x, \xi)\right)
$$

This gives the result, since $g$ is increasing.

(ii) Replace $\xi$ by $\vee_{i=0}^{n-1} T^{-i} \xi$ and $\eta$ by $\vee_{i=0}^{n-1} T^{-i} \eta$ in (i) and apply Definition 4.3 to get the result. 
The following theorem states the quantities in Definition 4.4 in terms of the $g$-entropy.

Theorem 4.7. Suppose that $T: X \rightarrow X$ is a continuous map on a compact metric space $X$ and $\mathcal{F} \subset[0,1]^{X}$ is the $\sigma$-algebra of Borel measurable maps $f: X \rightarrow[0,1]$. If $m \in M_{g}^{*}(X, T)$ and $m=\int_{E_{g}^{*}(X, T)} \nu \mathrm{d} \tau(\nu)$ is the $g$-ergodic decomposition of $m$ then

(i) If $\xi$ is a $g$-fuzzy partition then

$$
h_{m, g}^{*}(T, \xi)=\int_{E_{g}^{*}(X, T)} h_{\nu, g}(T, \xi) \mathrm{d} \tau(\nu) .
$$

(ii) If $\operatorname{card}\left(E_{g}^{*}(X, T)\right)<\infty$ then

$$
h_{m, g}^{*}(T)=\int_{E_{g}^{*}(X, T)} h_{\nu, g}(T) \mathrm{d} \tau(\nu) .
$$

Proof. (i) First, let $\nu \in E_{g}^{*}(X, T)$. By Lemma 3.1 (ii), $\mu_{\nu^{*}} \in E(X, T)$. By Birkhoff ergodic Theorem we have

$$
\begin{aligned}
\omega_{g}(T, x, f) & =g^{-1}\left(\limsup _{n \rightarrow \infty} \frac{1}{n} \sum_{k=0}^{n-1}(g \circ f) \circ T^{k}(x)\right) \\
& =g^{-1}\left(\int_{X} g \circ f \mathrm{~d} \mu_{\nu^{*}}\right) \\
& =g^{-1}\left(\nu^{*}(g \circ f)\right) \\
& =g^{-1} \circ \nu^{*} \circ g(f)
\end{aligned}
$$

for almost every $x \in X$. Since $\nu^{*}=g \circ \nu \circ g^{-1}$ we conclude that

$$
g\left(\omega_{g}(T, x, f)\right)=g(\nu(f))
$$

for almost every $x \in X$.

Therefore, if $\xi=\left\{f_{1}, f_{2}, \ldots, f_{k}\right\}$ is a $g$-fuzzy partition then

$$
\begin{aligned}
\Omega_{g}(T, x, \xi) & =g^{-1}\left(-\sum_{i=1}^{k} g\left(\omega_{g}\left(x, f_{i}\right)\right) \log g\left(\omega_{g}\left(x, f_{i}\right)\right)\right) \\
& =g^{-1}\left(-\sum_{i=1}^{k} g\left(\nu\left(f_{i}\right)\right) \log g\left(\nu\left(f_{i}\right)\right)\right. \\
& =H_{\nu, g}(\xi)
\end{aligned}
$$

for almost every $x \in X$.

For every $n \in \mathbb{N}$, replacing $\xi$ by $\bigvee_{i=0}^{n-1} T^{-i} \xi$, and considering the equality $g\left(\bigvee_{i=0}^{n-1} T^{-i} \xi\right)$ $=\bigvee_{i=0}^{n-1} T^{-i} g(\xi)$ we obtain

$$
\Omega_{g}\left(T, x, \bigvee_{i=0}^{n-1} T^{-i} \xi\right)=H_{\nu, g}\left(\bigvee_{i=0}^{n-1} T^{-i} \xi\right)
$$


for almost every $x \in X$ which simply results in

$$
\mathcal{H}_{g}(T, x, \xi)=h_{\nu, g}(T, \xi)
$$

for almost every $x \in X$. Integrating with respect to $\mu_{\nu^{*}}$ we obtain

$$
h_{\nu, g}^{*}(T, \xi)=h_{\nu, g}(T, \xi)
$$

for all $g$-fuzzy partitions $\xi$. Taking supremum over all $g$-fuzzy partitions we will have $h_{\nu, g}^{*}(T)=h_{\nu, g}(T)$.

Now, let $m \in M_{g}^{*}(X, T)$. For $n \geq 1$, let $f_{n}:=\min \left\{\mathcal{H}_{g}(T, \cdot, \xi), n\right\}$. Clearly, $\left\{f_{n}\right\}_{n=1}^{\infty}$ is an increasing sequence of bounded measurable functions such that $f_{n} \nearrow \mathcal{H}_{g}(T, \cdot, \xi)$. Applying Corollary 3.6, Monotone Convergence Theorem and (4) we will have

$$
\begin{aligned}
h_{m, g}^{*}(T, \xi) & =\int_{X} \mathcal{H}_{g}(T, x, \xi) \mathrm{d} \mu_{m^{*}}(x) \\
& =\lim _{n \rightarrow \infty} \int_{X} f_{n}(x) \mathrm{d} \mu_{m^{*}}(x) \\
& =\lim _{n \rightarrow \infty} \int_{E_{g}^{*}(X, T)}\left(\int_{X} f_{n}(x) \mathrm{d} \mu_{\nu^{*}}(x)\right) \mathrm{d} \tau(\nu) \\
& =\int_{E_{g}^{*}(X, T)}\left(\int_{X} \mathcal{H}_{g}(T, x, \xi) \mathrm{d} \mu_{\nu^{*}}(x)\right) \mathrm{d} \tau(\nu) \\
& =\int_{E_{g}^{*}(X, T)} h_{\nu, g}^{*}(T, \xi) \mathrm{d} \tau(\nu) \\
& =\int_{E_{g}^{*}(X, T)} h_{\nu, g}(T, \xi) \mathrm{d} \tau(\nu) .
\end{aligned}
$$

(ii) For $m \in E_{g}^{*}(X, T)$, let

$$
\mathfrak{D}_{m}:=\left\{\left\{\xi_{n}\right\}_{n \geq 1}: \xi_{n} \leq \xi_{n+1}, \quad h_{m, g}\left(T, \xi_{n}\right) \rightarrow h_{m, g}(T)\right\}
$$

then, by the supremum property, $\mathfrak{D}_{m} \neq \varnothing$. Also $\bigcap_{m \in E_{g}^{*}(X, T)} \mathfrak{D}_{m_{j}} \neq \varnothing$. To show this, let $E_{g}^{*}(X, T)=\left\{m_{1}, m_{2}, \ldots, m_{k}\right\}$. For each $j \in\{1,2, \ldots, k\}$ choose $\left\{\xi_{n}^{(j)}\right\}_{n \geq 1} \in \mathfrak{D}_{m_{j}}$. For $n \geq 1$, let $\xi_{n}:=\vee_{j=1}^{k} \xi_{n}^{(j)}$. For $j \in\{1,2, \ldots, k\}$, applying Theorem 4.6 (ii), we have

$$
h_{m_{j}, g}\left(T, \xi_{n}^{(j)}\right) \leq h_{m_{j}, g}\left(T, \xi_{n}\right) \leq h_{m_{j}, g}(T)
$$

Since $\left\{\xi_{n}^{(j)}\right\}_{n \geq 1} \in \mathfrak{D}_{m_{j}}$, the relation (5) results in

$$
\lim _{n \rightarrow \infty} h_{m_{j}, g}\left(T, \xi_{n}\right)=h_{m_{j}, g}(T)
$$

for all $j \in\{1,2, \ldots, k\}$. It means $\left\{\xi_{n}\right\}_{n \geq 1} \in \bigcap_{m \in E_{g}^{*}(X, T)} \mathfrak{D}_{m_{j}}$ which proves that $\bigcap_{m \in E_{g}^{*}(X, T)} \mathfrak{D}_{m_{j}} \neq \varnothing$. Now, we can choose a sequence $\left\{\xi_{n}\right\}_{n=1}^{\infty}$ of $g$-fuzzy partitions such 
that $\xi_{n} \leq \xi_{n+1} \quad(n=1,2, \ldots)$ and $\lim _{n \rightarrow \infty} h_{m, g}\left(T, \xi_{n}\right)=h_{m, g}(T)$ for all $m \in E_{g}^{*}(X, T)$. Applying part (i) and Monotone Convergence Theorem we will have:

$$
\begin{aligned}
h_{m, g}^{*}(T) & \geq \lim _{n \rightarrow \infty} h_{m, g}^{*}\left(T, \xi_{n}\right) \\
& =\lim _{n \rightarrow \infty} \int_{E_{g}^{*}(X, T)} h_{\nu, g}\left(T, \xi_{n}\right) \mathrm{d} \tau(\nu) \\
& =\int_{E_{g}^{*}(X, T)} \lim _{n \rightarrow \infty} h_{\nu, g}\left(T, \xi_{n}\right) \mathrm{d} \tau(\nu) \\
& =\int_{E^{*}(X, T)} h_{\nu, g}(T) \mathrm{d} \tau(\nu) .
\end{aligned}
$$

On the other hand

$$
h_{m, g}^{*}(T, \xi)=\int_{E_{g}^{*}(X, T)} h_{\nu, g}(T, \xi) \mathrm{d} \tau(\nu) \leq \int_{E_{g}^{*}(X, T)} h_{\nu, g}(T) \mathrm{d} \tau(\nu)
$$

for any given $g$-fuzzy partition $\xi$. This easily results in

$$
h_{m, g}^{*}(T) \leq \int_{E_{g}^{*}(X, T)} h_{\nu, g}(T) \mathrm{d} \tau(\nu)
$$

which completes the proof.

\section{SUMMARY AND CONCLUSIONS}

This paper is devoted to a local study of the concept of $g$-entropy of dynamical systems. The set of $g$-invariant and $g$-ergodic fuzzy measures is defined in section 3 . It is equipped to a weak* topology such that the set of $g$-invariant fuzzy measures is the convex hull of the set of $g$-ergodic fuzzy measures. Then the $g$-ergodic decomposition is introduced. A new type of $g$-entropy is defined in section 4 . This definition is of local entity. Using the framework constructed in section 4 , the new quantity is stated in terms of the known $g$-entropy.

\section{ACKNOWLEDGEMENT}

The author would like to thank the referees for their comprehensive and useful comments which helped the improvement of this work to the present form.

(Received June 8, 2014)

\section{REFERENCES}

[1] M. Brin and A. Katok: On local entropy in geometric dynamics. SpringerVerlag, New York 1983 (Lecture Notes in Mathematics 1007) (1983), pp.30-38. DOI:10.1007/bfb0061408 
[2] D. Butnariu and E.P. Klement: Triangular norm-based measures and their Markov kernel representation. J. Math. Anal. Appl. 162 (1991), 111-143. DOI:10.1016/0022247x(91)90181-x

[3] D. Dumitrescu: Measure-preserving transformation and the entropy of a fuzzy partition. In: 13th Linz Seminar on Fuzzy Set Theory, Linz 1991, pp. 25-27.

[4] D. Dumitrescu: Fuzzy measures and the entropy of fuzzy partitions. J. Math. Anal. Appl. 176 (1993), 359-373. DOI:10.1006/jmaa.1993.1220

[5] D. Dumitrescu: Entropy of a fuzzy process. Fuzzy Sets and Systems 55 (1993), 169-177. DOI:10.1016/0165-0114(93)90129-6

[6] D. Dumitrescu: Entropy of fuzzy dynamical systems. Fuzzy Sets and Systems 70 (1995), 45-57. DOI:10.1016/0165-0114(94)00245-3

[7] D. Markechová: The entropy on F-quantum spaces. Math. Slovaca 40 (1990), 177-190.

[8] D. Markechová: The entropy of fuzzy dynamical systems and generators. Fuzzy Sets and Systems 48 (1992), 351-363. DOI:10.1016/0165-0114(92)90350-d

[9] D. Markechová: Entropy of complete fuzzy partitions. Math. Slovaca 43 (1993), 1, 1-10.

[10] D. Markechová: A note to the Kolmogorov-Sinaj entropy of fuzzy dynamical systems. Fuzzy Sets and Systems 64 (1994), 87-90. DOI:10.1016/0165-0114(94)90009-4

[11] B. McMillan: The basic theorems of information theory. Ann. Math. Statist. 24 (1953), 196-219. DOI:10.1214/aoms/1177729028

[12] R. Mesiar and J. Rybárik: Entropy of fuzzy partitions: A general model. Fuzzy Sets and Systems 99 (1998), 73-79. DOI:10.1016/s0165-0114(97)00024-9

[13] M. Rahimi and A. Riazi: Entropy operator for continuous dynamical systems of finite topological entropy. Bull. Iranian Math. Soc. 38 (2012), 4, 883-892.

[14] M. Rahimi and A. Riazi: On local entropy of fuzzy partitions. Fuzzy Sets and Systems 234 (2014), 97-108. DOI:10.1016/j.fss.2013.02.006

[15] B. Riečan: On a type of entropy of dynamical systems. Tatra Mountains Math. Publ. 1 (1992), 135-140.

[16] B. Riečan: On the g-entropy and its Hudetz correction. Kybernetika 38 (2002), 4, 493-500. DOI:10.1016/j.physa.2007.06.047

[17] B. Riečan and D. Markechová: The entropy of fuzzy dynamical systems, general scheme and generators. Fuzzy Sets and Systems 96 (1998), 191-199. DOI:10.1016/s01650114(96)00266-7

[18] B. Riečan and T. Neubrunn: Integral, Measure, and Ordering. Kluwer, Dordrecht and Ister, Bratislava 1997. DOI:10.1007/978-94-015-8919-2

[19] J. Rybárik: The entropy of the Q-F-dynamical systems. Busefal 48 (1991), 24-26.

[20] J. Rybárik: The entropy based on pseudoarithmetical operations. Tatra Mountains Math. Publ. 6 (1995), 157-164.

[21] Ya. Pesin: Characteristic Lyapunov exponents and smooth ergodic theory. Russian Math. Surveys 32 (1977), 54-114. DOI:10.1070/rm1977v032n04abeh001639

[22] R. Phelps: Lectures on Choquet's Theorem. Van Nostrand, Princeton, N.J. 1966.

[23] D. Ruelle: An inequality for the entropy of differential maps. Bol. Soc. Bras. de Mat. 9 (1978), 83-87. DOI:10.1007/bf02584795 
[24] P. Walters: An Introduction to Ergodic Theory. Springer-Verlag, 1982.

Mehdi Rahimi, Department of Mathematics, Faculty of Science, University of Qom, Qom. Iran.

e-mail: m10.rahimi@gmail.com 